\author{
Paulo R. Aguiar \\ aguiarpr@feb.unesp.br \\ Paulo J. A. Serni \\ paulojas@feb.unesp.br \\ Fábio R. L. Dotto \\ dotto@ladaps.feb.unesp.br \\ Electrical Engineering Department - UNESP \\ 17033-360 Bauru, SP, Brazil
}

\author{
Eduardo C. Bianchi \\ Senior Member, ABCM \\ bianchi@feb.unesp.br \\ Mechanical Engineering Department - UNESP \\ 17033-360 Bauru, SP, Brazil
}

\section{In-Process Grinding Monitoring Through Acoustic Emission}

This work aims to investigate the efficiency of digital signal processing tools of acoustic emission signals in order to detect thermal damages in grinding processes. To accomplish such a goal, an experimental work was carried out for 15 runs in a surface grinding machine operating with an aluminum oxide grinding wheel and ABNT 1045 Steel as work material. The acoustic emission signals were acquired from a fixed sensor placed on the workpiece holder. A high sampling rate data acquisition system working at $2.5 \mathrm{MHz}$ was used to collect the raw acoustic emission instead of the root mean square value usually employed. Many statistical analyses have shown to be effective to detect burn, such as the root mean square (RMS), correlation of the $A E$, constant false alarm rate (CFAR), ratio of power $(R O P)$ and mean-value deviance $(M V D)$. However, the CFAR, ROP, Kurtosis and correlation of the AE have been presented more sensitive than the RMS.

Keywords: Grinding, burn detection, acoustic emission, electrical power, monitoring

\section{Introduction}

The market revolution in the last years has been demanded from industries effective cost reduction in connection with quality increase of the parts manufactured by machining operations. This built-up need faces increasingly to the manufacturing problems, such as rapid setup and the wear of the cutting tools (Dorfeld, 1994). Moreover, due to changes in the production system characteristics, that is, increase of the small batches and high costs of qualified personnel, the use of production systems fully flexible and automatic, capable of compensating the process variations became imperative. This implies in system developing and strategies for the process monitoring in a manner that the automation with safety and repeatability can be achieved. The grinding process is not technologically known enough yet. This may come from the wrong belief in which the process is very complex to be understood due to the multiplicity of cutting edges and their irregular geometries, high cutting speed and depth of cut that varies from grain to grain. Damages in the part are very expensive, since all the previous processes before the grinding itself are wasted when a part is scrapped at this stage. The need of effective reduction of costs associated with the increase of quality of the parts manufactured claim the implementation of more intelligent systems in the industrial environments. Hence, the damage control in grinding process is of great interest to every industry dependent on this process, leading to lower scrap rates and, in turn, to a lower production cost. Thus, the present work aims to investigate new statistical tools to detect burn in grinding by digitally processing the acoustic emission signals generated during the process.

\section{Monitoring And Process Control}

The implementation of intelligent processes in industries utilizing computer numerically controlled machining is increasing rapidly. However, computer numerically controlled systems are not reliable enough to operate without human interference. It is usual to observe operators of these CNC machines simply correcting the process parameters or identifying the end of tool life (Aguiar et al, 1999). According to Inasaki (1989), there are three important goals to the grinding process monitoring: detecting problems, which occur during the process; providing information to optimize the process

Paper accepted October, 2005. Technical Editor: Anselmo Eduardi Diniz. and contributing to the development of a database needed to determine the control parameters. Taking as an example the external plunge grinding process, there are many parameters which need to be pre-determined, which are related to the choice of the grinding wheel and cutting fluid. The wheel speed, work speed and feed rate are the relevant parameters. Among these parameters, the feed rate is the most important affecting the grinding results. The choice of the grinding cycle that consists on determining the desired roughness, the end of the operation and spark-out period are other important parameters to be considered. The information obtained during the system monitoring may be used to minimize the grinding cycle time and increase the process quality (Inasaki, 1989). The use of acoustic emission (AE) to monitor and control the grinding process is a relatively recent technology (Bennett, 1994; Aguiar, 2003), besides being more sensitive to the grinding condition variations compared with the force and power measurements (Webster, 1994, 1996), providing a promising technique to the process monitoring. The relatively easiness of digitally processing the root mean square (RMS) of the acoustic emission signal has led to approaches in which this type of statistic is employed. However, the inherent typical operation involved in determining the RMS of acoustic emission signal makes it to a certain extent insensitive to impulsive events such as cracks and burn on the part, although this kind of parameter carries a lot of useful information (Benett, 1994). In this present work, tests results are presented for ABNT 1045 steel and aluminum oxide grinding wheel; setup employed; and tests offline to evaluate the superficial integrity of the parts ground. Furthermore, results from many digital signal processing tools are shown, where those with amplitude independent are stressed and then the power of the $\mathrm{AE}$ signal does not affect the signal characteristic. This is explained due to the fact that the power of the AE signal may undergo variations during the grinding process, which have nothing to do with the part condition than its geometry. The result analyses from the digital signal processing as well as a discussion of the investigation are presented.

\section{Experimental Tests}

The experimental tests were carried out upon a surface grinding machine where the raw acoustic emission signal was collected for seven (7) different runs at $2.5 \mathrm{MHz}$. The ABNT 1045 steel was used for the tests. The major parameters were kept constant during the runs. However, the depth of cut was varied from light to aggressive cutting. All the parts were essayed after the grinding tests and the 
burn marks were identified. The setup for these runs is shown in Figure 1.

The grinding parameters include: grinding wheel peripheral speed: $27.94 \mathrm{~m} / \mathrm{s}$; workpiece speed: $0.044 \mathrm{~m} / \mathrm{s}$; cutting fluid: waterbased fluid 4\%; grinding wheel: aluminum oxide - 38a80-pvs Norton; grinding wheel diameter: $296.50 \mathrm{~mm}$; grinding wheel width: $40.21 \mathrm{~mm}$; workpiece dimensions: 98.58 x $8.74 \mathrm{~mm}$.

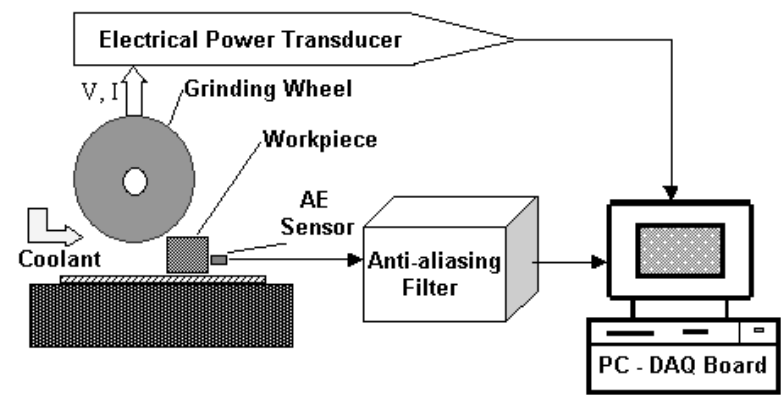

Figure 1. Experimental Setup.

Data was collected from a fixed acoustic emission sensor from Sensis, model PAC U80D-87, which was mounted on the part holder. The data acquisition board from National Instruments was set up to work at $2.5 \mathrm{MHz}$ with a 12 bits precision per sample. Table 1 shows details of the tests carried out for the ABNT 1045 steel.

Table 1. Tests with ABNT 1045 Steel.

\begin{tabular}{|c|c|c|c|c|}
\hline Test & \multicolumn{2}{|c|}{ Depth of cut $[\boldsymbol{\mu m}]$} & Cutting Profile & Comments \\
\hline 1 & 10 & & $\square$ & No burn \\
\hline 2 & 30 & & $\square$ & Slight burn \\
\hline 3 & 20 & & $\square$ & Severe burn \\
\hline 4 & 90 & 10 & $\square--$ & Severe burn \\
\hline 5 & 20 & 2,5 & $\square--$ & Severe burn \\
\hline 6 & 40 & 5 & $\square$ & $\begin{array}{c}\text { Severe burn } \\
\text { Burn at } \\
\text { middle }\end{array}$ \\
\hline 7 & 15 & & $\square$ & \\
\hline
\end{tabular}

The two columns showed in Table 1 which represent the depth of cut indicate the depth of cut in the beginning and in the end of the grinding pass respectively. When the second column is blank means the depth of cut was kept constant along the grinding pass and equal to the number indicated in the first column. That also explains the column 'Cutting Profile' where a sketch of the workpiece was placed accordingly.

From the workpieces tested, a visual inspection was carried out in which visible burn marks were observed by different color patterns on the surface of the workpieces. These colorations varies from grey to black, depending on the burn level occurred, according to Malkin (1989). The workpiece pictures are shown in Figure 2.

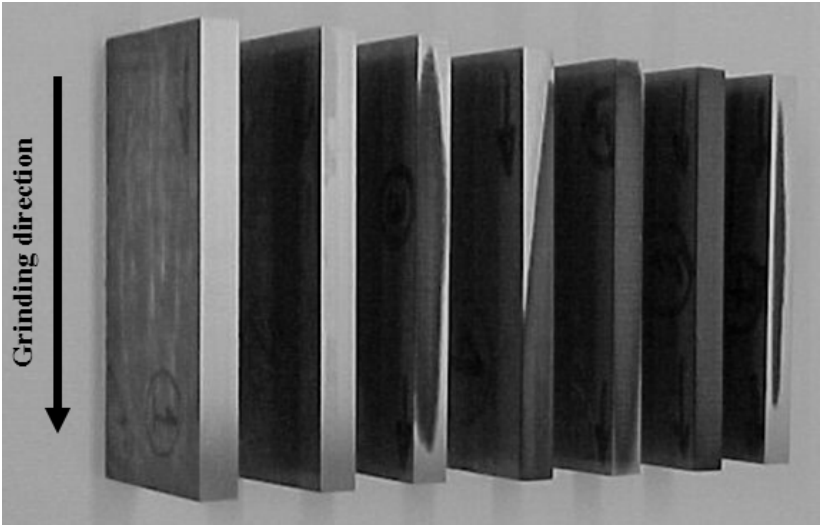

Figure 2. Workpieces from tests 1 to 7 from left to right.

Besides the visual analysis, surface roughness and microhardness tests were performed on the workpieces tested to better characterize the workpiece burn. Figure 3 shows the results for the roughness tests which was carried out with a portable Taylor Hobson Surtronic 3, and Figure 4 for the microhardness tests performed with the device Otto Wolpert-Werke, type M-Testor, Baujahr.

It can be seen in Figure 3 that the surface roughness values are higher for the workpieces where burn took place. Thus, the surface roughness may be considered a parameter to indicate the existence of burn on the workpiece, since it provokes a metallurgical transformation of the part and in turn a loss of its surface quality with a possible softening and sudden hardening of the material in certain regions.
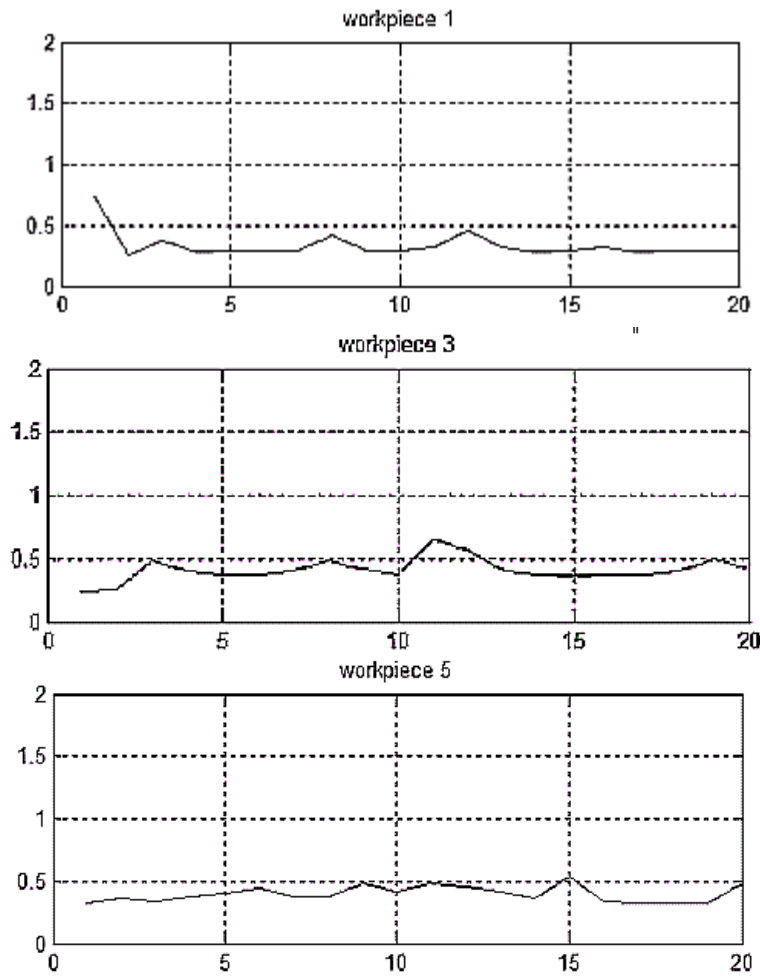

Figure 3. Surface roughness $(\mu \mathrm{m})$ for workpieces 1 to 7 . The horizonta axis represents the measurements taken each $5 \mathrm{~mm}$ along the workpieces. 

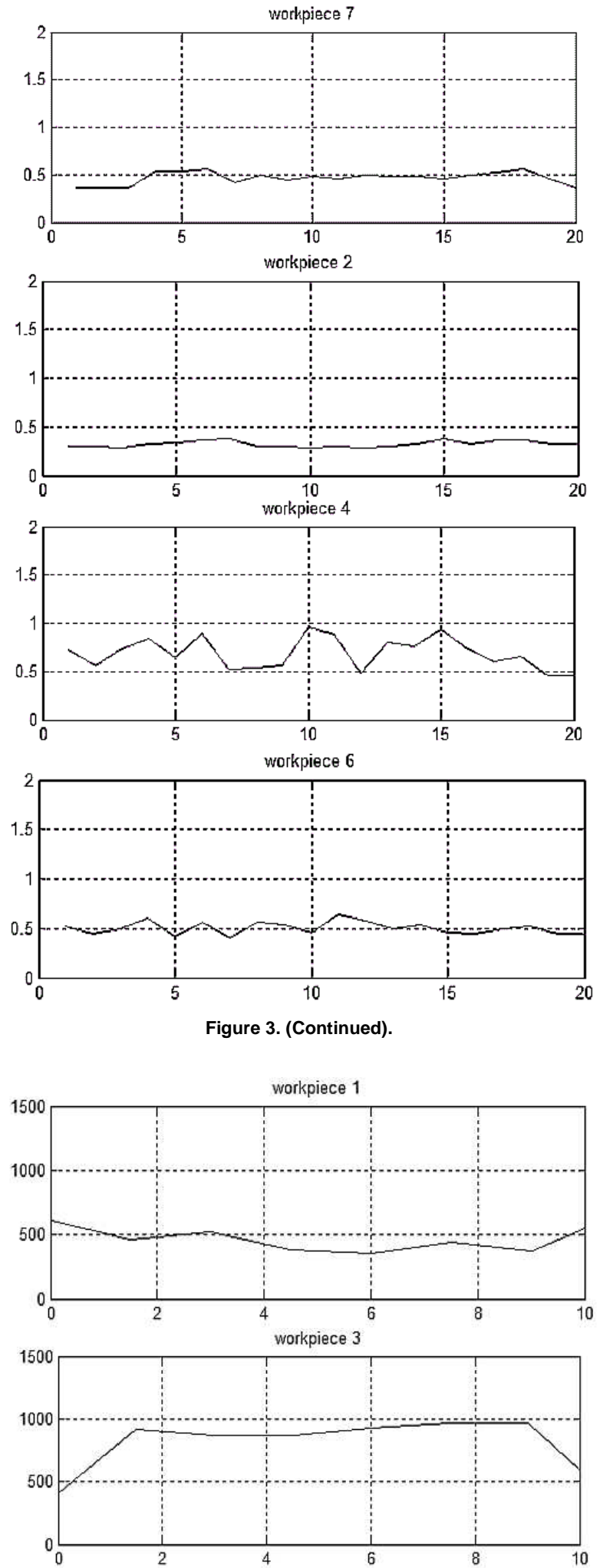

Figure 4. Microhardness (Vickers) for workpieces 1 to 7 . The horizonta axis represents the measurements taken each $15 \mathrm{~mm}$ along the workpiece, except the last one.
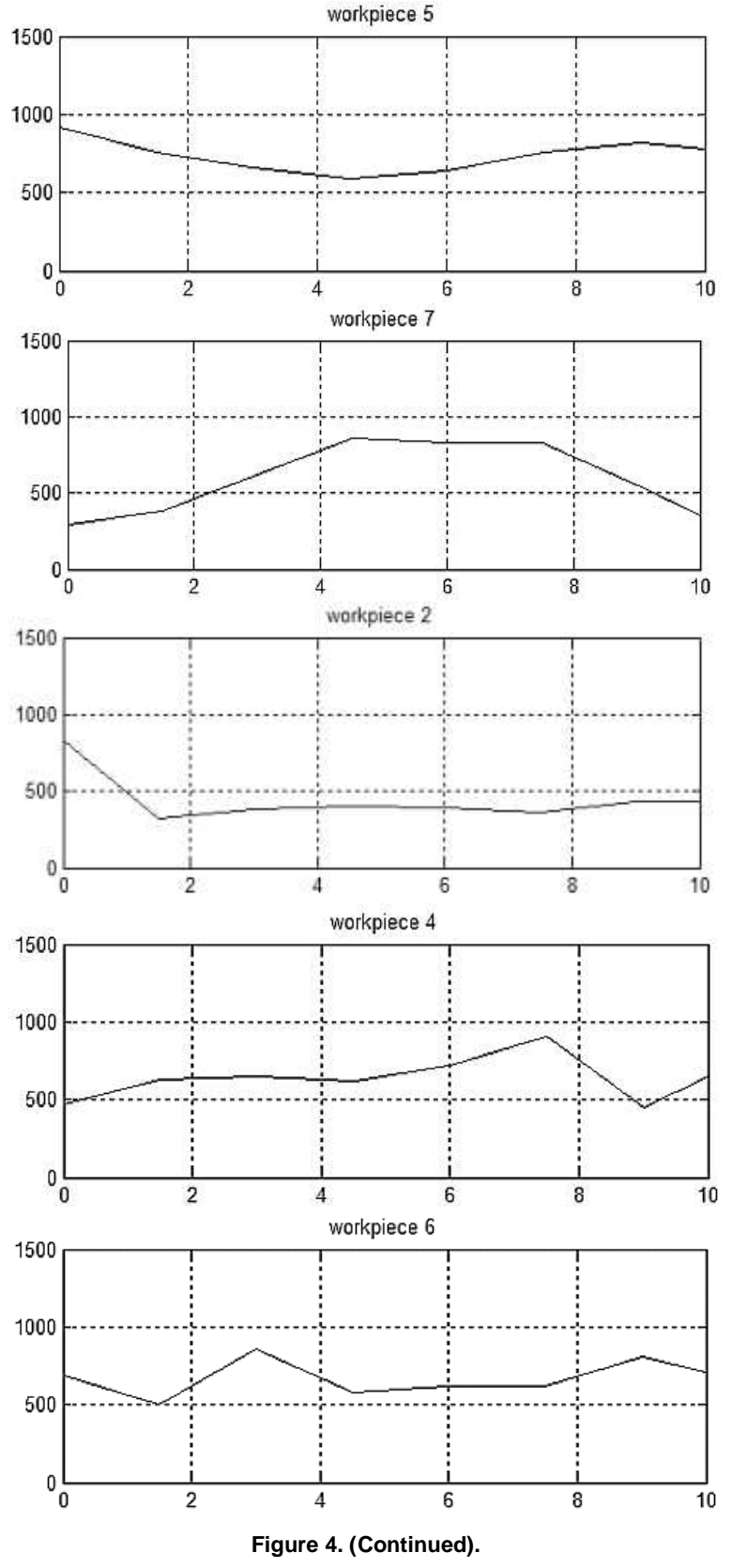

Figure 4 clearly shows the hardening and softening phenomenon occurred in the material when burn or non-burn takes place during the grinding process. It can be observed for workpiece 1 which did not undergo burn, and also for workpiece 2 which underwent slight burn, the microhardness levels have not presented significant variations along the workpiece. On the other hand, the level of the hardness has considerably varied for those workpieces that underwent severe burn.

Therefore, a better analysis of the workpieces tested regarding the burn occurrence is possible based on the surface roughness and microhardness results.

\section{Signal Processing}

From the data of acoustic emission available on binary files, several programs developed using Matlab for digitally processing the signals were utilized, where many statistical correlations such as 
kurtosis, skew, autocorrelation, RMS among others were employed and are described as following.

\section{RMS of Acoustic Emission Signal}

For a given time $\mathrm{t}$, the RMS value of a raw acoustic emission signal can be expressed by

$$
A E_{r m s}=\sqrt{\frac{1}{T} \int_{t-T}^{t} A E_{r m s}^{2}(\tau) d \tau}=\sqrt{\frac{1}{N} \sum_{i=1}^{N} A E_{r m s}^{2}(i)}
$$

where $\mathrm{T}$ is the integration time and $\mathrm{N}$ is the discrete number of $\mathrm{AE}$ data within the interval $\mathrm{T}$. In this work, $\mathrm{T}$ was considered equal to 1 ms (Webster, 1996).

\section{Constant False Alarm Rate - CFAR}

Nuttal (1999) is a statistic tool employed in detection of events, which is described by

$$
T_{p l}(x)=\sum_{k=0}^{M-1} X_{k}^{v}
$$

Where $\mathrm{Xk}$ corresponds to the $\mathrm{kth}$ FFT of $\mathrm{x}(\mathrm{t}), \mathrm{v}$ is a variable exponent and $2 \mathrm{M}$ corresponds to the data base vector size to get the FFT calculated. Although $v$ between 2 and 3 provides a satisfactory performance for a wide band of frequency of the signal studied, this statistic needs of pre-normalized data. Thus, due to the acoustic emission signal variations during the process, the constant false alarm rate (CFAR) is utilized, (Nuttal, 1994). This parameter is based on the supposition of flatness of the acoustic emission signal. An alternative version of this tool was employed due to the system distortions and expressed by the equation 3 (Wang, 1999).

$$
T_{b c p l}(X)=\frac{\sum_{k=n l}^{n 2} X_{k}^{v}}{\sum_{n 1}^{n 2} X_{k}}
$$

Were $M=1280$ and the frequency range between 300 and 700 $\mathrm{kHz}$ was considered.

\section{Kurtosis and Skewness}

The measurement if the distribution tail is longer than other is made by Skew. In case of kurtosis, the tail size is concerned. Both parameters are utilized in this work aiming to find an indicator to the acoustic emission variations. Thus, abrupt changes in the $\mathrm{AE}$ signal such as those in which burn occurs may result in spikes in these parameters. Equations 4 and 5 show how to calculate kurtosis and skewness of an $\mathrm{x}$ signal.

$$
\begin{gathered}
K=\sum \frac{(x-\mu)^{4}}{N \sigma^{4}}-3 \\
K=\sum \frac{(x-\mu)^{3}}{N \sigma^{3}}
\end{gathered}
$$

where $\mu$ is the mean of $x, N$ the number of samples in the range considered and $\sigma$ the standard deviation.

\section{Mean Value Dispersion Statistic - MVD}

The form of MVD statistic is therein employed (Chen et al, 1999) but in a more convenient form used by Wang (1999) as shown in equation 6.

$$
M V D=\sum_{k=n 1}^{n 2} \log \left(\frac{\frac{1}{n_{2}-n_{1}+1} \sum_{l=n 1}^{n 2} X_{l}}{X_{k}}\right)
$$

Where $\mathrm{X}$ has the same meaning as to CFAR statistic as well as $\mathrm{n}_{1}$ e $\mathrm{n}_{2}$.

\section{Ratio of Power - ROP}

It is instinctive consider that different behaviors can be expected for a good part or bad one by observing the frequency spectrum of the acoustic emission signal. Hence, for each block of acoustic emission data ROP is given by equation 7 .

$$
R O P=\sum_{k=n_{1}}^{n_{2}} \frac{\left|X_{k}\right|^{2}}{\sum_{k=0}^{N-1}\left|X_{k}\right|^{2}}
$$

where $\mathrm{N}$ set to $1024 ; \mathrm{n} 1$ and $\mathrm{n} 2$ in the range of 300 to $700 \mathrm{kHz}$ were chosen.

\section{Autocorrelation}

The time correlation of a function $\Phi_{\mathrm{xy}}$ is defined by Oppenheim (1997) in Equation 8.

$$
\phi_{x y}(t)=\int_{-\infty}^{+\infty} x(t+\tau) y(\tau) d \tau
$$

$\Phi_{\mathrm{xx}}$ is commonly referred to as autocorrelation.

\section{Results and Discussion}

The graphs for each workpiece tested were obtained from the digital signal processing of acoustic emission signals in which the statistics previously described were employed. The results from tests 1, 2, 4 and 7 for ABNT 1045 steel are presented as shown in Figures 5 to 8 , respectively. The results from other tests are not presented due to the similarities to these shown here.

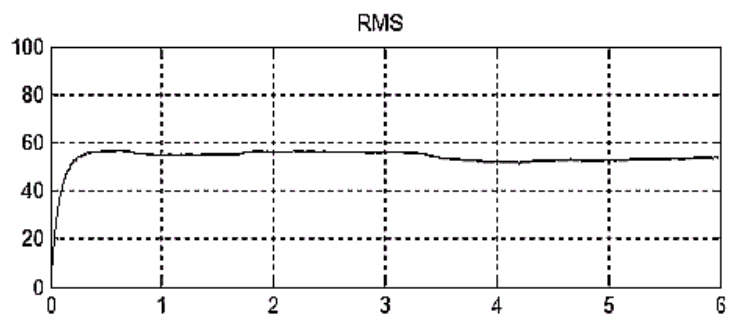

Figure 5. Results for test 1 - with no burn occurrence; (horizontal axis corresponds time in seconds and vertical axis Volts multiplied by a constant). 

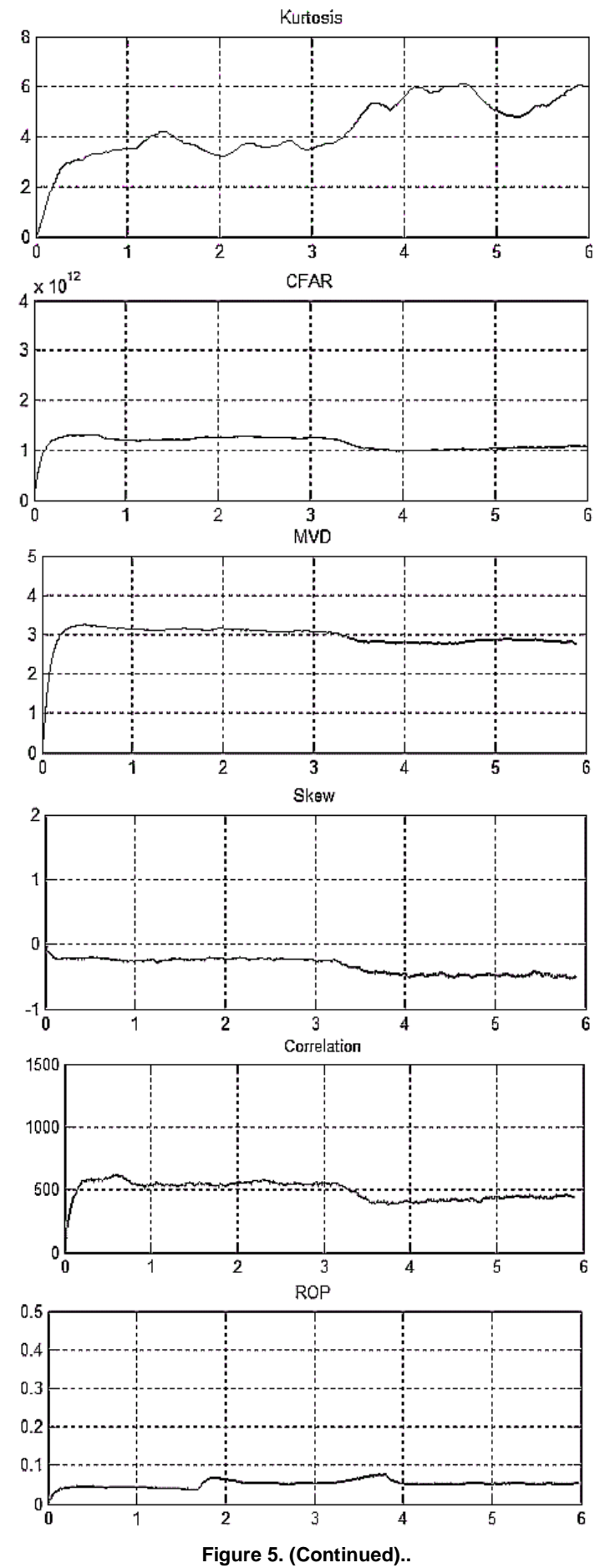

Figure 5. (Continued)..
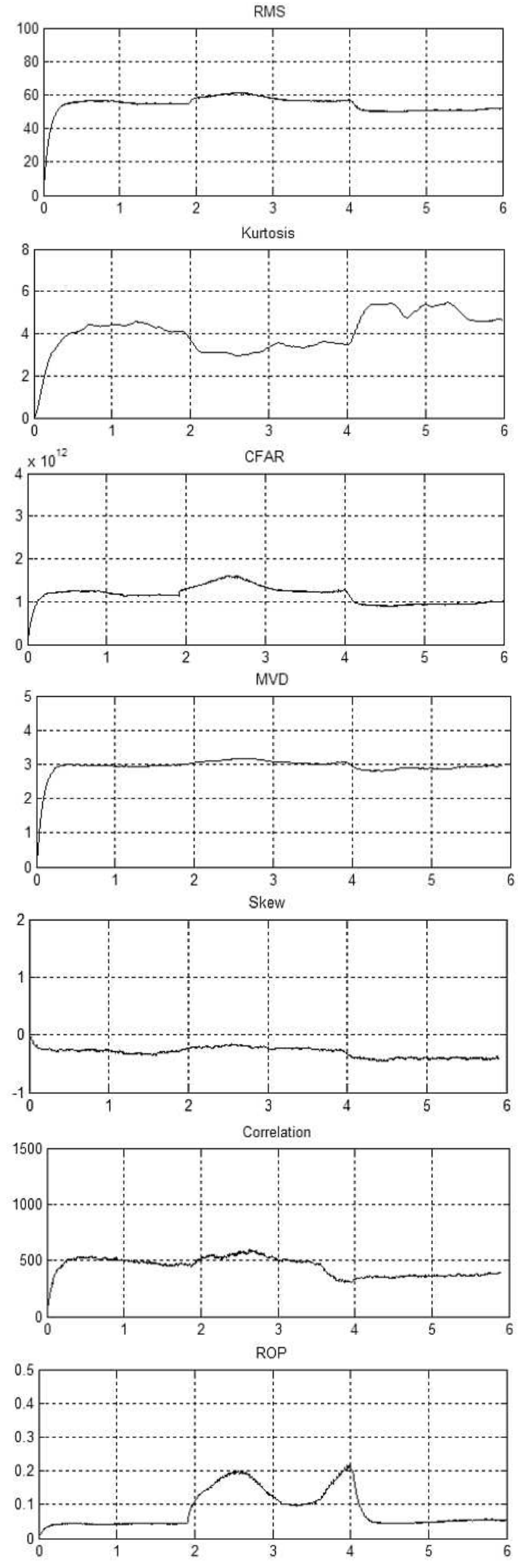

Figure 6. Results for test 2 -with slight burn occurrence; (horizontal axis corresponds time in seconds and vertical axis Volts multiplied by a constant). 

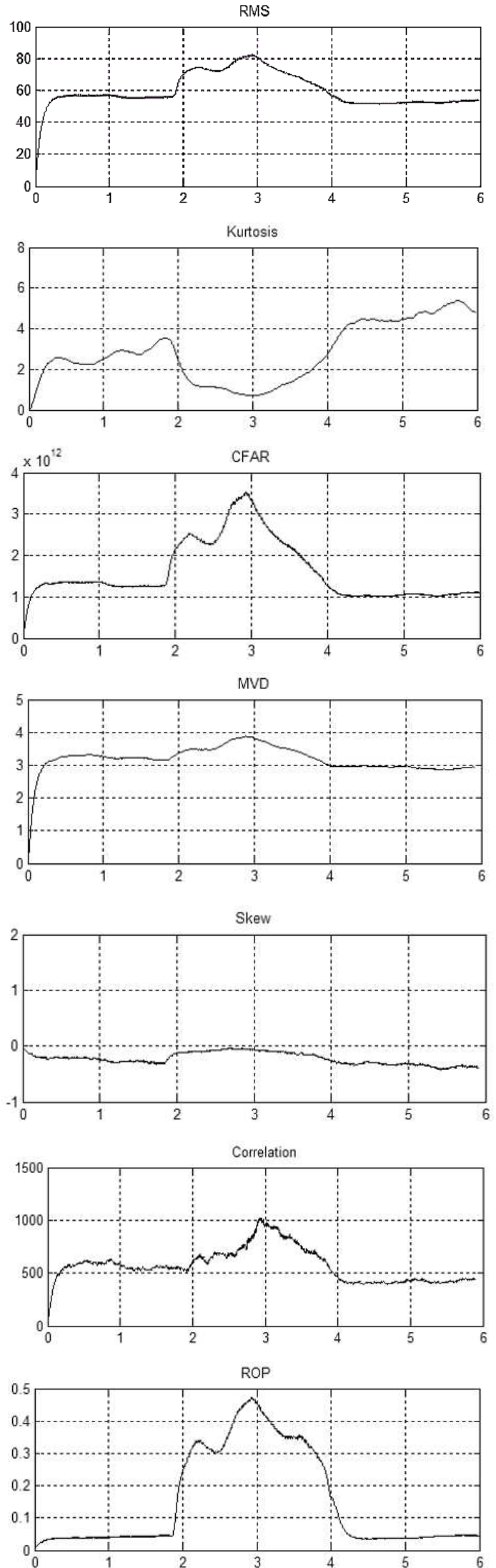

Figure 7. Results for test 4 -with severe burn occurrence; (horizontal axis corresponds time in seconds and vertical axis Volts multiplied by a constant).

J. of the Braz. Soc. of Mech. Sci. \& Eng.
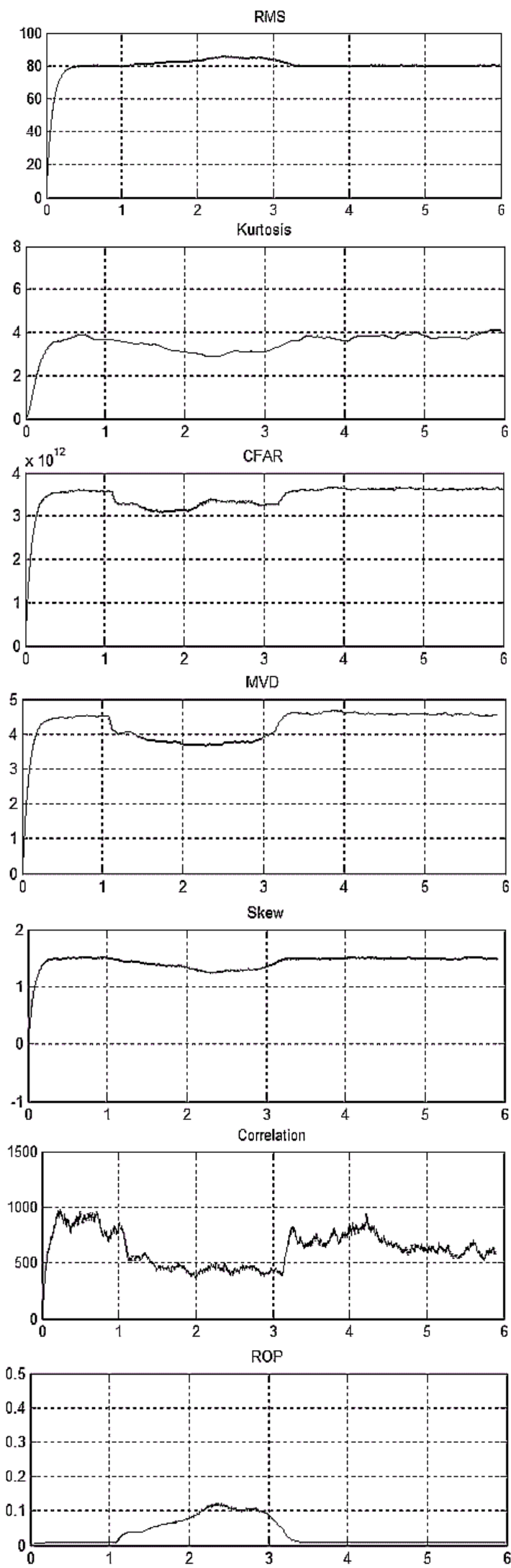

Figure 8. Results for test 7 -with severe burn occurrence; (horizontal axis corresponds time in seconds and vertical axis Volts multiplied by a constant). 
From the results for the ABNT 1045 steel it can be observed that the RMS value had its level quite steady for the non-burning workpiece during the grinding pass while the signal had good variation when severe burn occurred in test 4 and test 7, Figure 7 and 8 respectively. However, the level did not presented a significant change when the slight burn occurred, as in test 2, Figure 6.

Skewness and kurtosis presented variation when burn took place but positive amplitudes for some tests and negative ones for others were observed, which are not useful for an indicator parameter to burn.

The autocorrelation of the acoustic emission signal was sensitive for most of the tests carried out. For test 1 with no burn, Figure 5, the level of this statistic has kept stead during the grinding pass while for other tests when burn occurred the level has increased, except for test 7, Figure 8. Thus, this parameter is not useful to burn detection.

Similarly to the autocorrelation, the CFAR tool has behaved quite well to burn detection for most of the tests performed but with no decreasing of signal, except for test 7 , Figure 8 , where a decreasing was observed during the grinding pass. This behavior, however, did not compromise the utility of CFAR tool, for the level of test 7 has kept higher than to the non-burning test. In addition, the results for this tool have clearly shown to be more sensitive to burn detection than the RMS parameter, for the levels have presented more variations besides being higher. The CFAR levels seem to give more information about the location of burn.

Surprisingly, the ROP statistic turned out to be a good indicative to burn, since its behavior has shown to be quite sensitive to the studied phenomenon. Besides, its level is lower to those non-burned parts and higher to the burned ones. Additionally, it has well characterized the beginning of contact between the wheel and part as well as the end of the grinding pass. In a similar way to the CFAR statistic, the ROP tool has presented a very good variation when burn took place and it seems to give more information about the location of burn, then showing up more sensitive than the RMS values.

The MVD statistic has shown variations similar to the RMS value. Although a decrease of the signal level can be observed in test 7 with severe burn in the middle of the workpiece, Figure 8 , it is still a good burn indicator because this level is higher than to the non-burned workpiece, as in test 1 , Figure 5, and to the slight burned workpiece, test 2, Figure 6.

It can be observed from the results that only the RMS value and CFAR statistic have presented sensitive to detect slight burn effectively.

\section{Conclusions}

From digital processing of the raw acoustic emission signal for the ABNT 1045 steel, the results show that several statistics have worked satisfactory to burn detection as is the case of RMS, CFAR,
ROP e MVD. It can be clearly seen that the CFAR and ROP statistics have presented more variation of their levels when burn took place than the RMS value. In addition, these statistics may be better explored in other studies because they seem to give more information about the location of the burn and surface profile. Nevertheless, skew and kurtosis statistics have presented an interesting behavior regarding the waveform of the signal and their variation along the grinding pass, though they are not effective to detect burn. These features may be better explored in a further investigation. The autocorrelation has shown ineffective to detect burn.

\section{Acknowledgments}

The authors want to express their appreciation to Fundação de Amparo à Pesquisa do Estado de São Paulo - FAPESP and IFM Institute Factory of Millennium, for their support given to this work which became viable its development.

\section{References}

Aguiar, P. R, Willet, P. and Webster, J., 1999, "Acoustic Emission Applied to Detect Workpiece Burn During Grinding", International Symposium on Acoustic Emission: Standards and Technology Update, S. Vahaviolos, Ed., Fort Lauderdale, FL., Vol. STP 13, pp. 107-124.

Aguiar, P. R., 2003, "Automatic Control of Thermal Damages in Grinding by Acoustic Emission and Electric Power Signals", (In Portuguese), Thesis of Associate Professor at Sao Paulo State University Unesp, Bauru Campus, Sao Paulo State, 204p.

Bennett, R. T., 1994, “Acoustic Emission in Grinding”, Master Thesis, University of Connecticut.

Chen, B., Willett, P. and Streit, R., 1999, "Transient Detection using a Homogeneity Test", The 1999 IEEE Conference on Acoustics, Speech and Signal Processing, Phoenix, AZ, pp. 2769-2772.

Dornfeld, D., 1994, "In Process Recognition of Cutting States", JSME International Journal, volume 37, n 4, pp638-650.

Inasaki, I., 1989, "Sensor Fusion for Monitoring and Controlling Grinding Processes", The International Journal of Advanced Manufacturing Technology, pp. 730-736.

Malkin, S., 1989, "Grinding Technology: Theory and Applications of Machining Abrasives", England: Ellis Horwood Limited, 275p.

Oppenheim, A. V. And Willsky, A. S., 1997, "Signals \& Sistems", 2sd. Edition, Prentice Hall Signal Processing Series.

Nuttal, A. 1994 "Detection Performance of Power-Law Processors for Random Signals of Unknown Location, Structure, Extent, and Strength", Naval Undersea Warfare Center Technical Report 10,751.

Nuttal, A., 1999, "Performance of Power-Law Proessors with Normalization for Random Signals of Unknown Structure”, Naval Undersea Warfare Center, NPT Technical Report 10,760.

Wang, Z., 1999, "Surface Grinding Monitoring by Signal Processing of Acoustic Emission Signals", Master Thesis, University of Connecticut.

Webster, J., Marinescu, I. and Bennet, R, 1994, "Acoustic Emission for Process Control and Monitoring of Surface Integrity during Grinding", Annals of the CIRP, volume 43/1, p. 299-304.

Webster J, Dong, W. and Lindsay, R., 1996, "Raw Acoustic Emission Signal Analysis of Grinding Process", Annals of the CIRP, Vol 45/1. 